\title{
Cambios hemodinámicos y calidad de vida en pacientes con cáncer de próstata luego de un entrenamiento HIIT Y MICT. Ensayo clínico aleatorizado (ONCO-EXE TRIAL)
}

\section{Hemodynamic changes and quality of life in patients with prostate cancer after a HIIT and MICT training. A randomized clinical trial (ONCO-EXE TRIAL)}

\author{
Javier Eliecer Pereira-Rodríguez ${ }^{1, a, b, c}$, Devi Geesel Peñaranda-Florez ${ }^{2, d e}$, Ricardo Pereira-Rodríguez ${ }^{3, f}$, Pedro Pereira-Rodríguez ${ }^{4, g}$, \\ Karla Noelly Santamaría-Perez ${ }^{1, h}$ \\ ${ }^{1}$ Universidad Tolteca de México. Puebla, México. \\ ${ }^{2}$ Investigador independiente. Puebla, México. \\ ${ }^{3}$ Fundación Universitaria de Ciencias de la Salud. Bogotá, Colombia. \\ ${ }^{4}$ Coorporación Universitaria Rafael Núñez. Cúcuta, Colombia. \\ ${ }^{a}$ Fisioterapeuta, especialista en rehabilitación cardiopulmonar \\ ${ }^{\mathrm{b}}$ Maestrante en ciencias de la salud \\ 'Maestrante en innovación educativa \\ ${ }^{\mathrm{d}}$ Fisioterapeuta, especialista en neurorehabilitación \\ ${ }^{e}$ Magister en dificultades del aprendizaje \\ ${ }^{\dagger}$ Médico residente en medicina de urgencias y cuidado del paciente en estado crítico \\ ${ }^{9}$ Médico general \\ ${ }^{\mathrm{h}}$ Estudiante de fisioterapia
}

An Fac med. 2019;80(4):419-26 / DOI: https://doi.org/10.15381/anales.v80i4.16218

\section{Correspondencia: \\ Javier E. Pereira Rodríguez \\ jepr87@hotmail.com}

Recibido: 29 de mayo 2019

Aceptado: 25 de setiembre 2019

Publicación en línea: 28 de diciembre 2019

Conflictos de interés: Los autores declaran no tener conflictos de interés.

Fuente de financiamiento: Autofinanciado

Contribuciones de autoría: JPR y DPF participaron en la concepción y diseño del estudio. DPF y PPR participaron en el diseño y las intervenciones del estudio. RPR y PPR participaron en la recolección de los datos. KSP participó en la tabulación de los datos y análisis estadístico. Todos los autores participaron en la interpretación y análisis de los resultados. Todos los autores redactaron el artículo y aprobaron la versión final. Los autores pertenecen al grupo de investigación Aletheia.

Citar como: Pereira-Rodriguez JE, Peñaranda-Florez DG, Pereira-Rodríguez $R$, Pereira-Rodriguez $P$, Santamaría-Perez KN. Cambios hemodinámicos y calidad de vida en pacientes con cáncer de próstata luego de un entrenamiento HIIT Y MICT. Ensayo clínico aleatorizado (ONCOEXE TRIAL). An Fac med. 2019;80(4):41926. DOI: https:/doi.org/10.15381/anales. v80i4.16218

\section{Resumen}

Introducción. El cáncer de próstata es el más frecuente en hombres y constituye la segunda causa de mortalidad. La rehabilitación basada en ejercicios se muestra efectiva y segura para mitigar los efectos del cáncer. Objetivo. Determinar los cambios hemodinámicos y calidad de vida en pacientes con cáncer de próstata luego de un entrenamiento de alta intensidad (HIIT) o continuo, a intensidad moderada (MICT). Métodos. Ensayo clínico aleatorizado con 249 pacientes con cáncer de próstata en estadio II distribuidos en tres grupos (MICT, HIIT y grupo control) a quienes se les aplicó el cuestionario EORTC QLQ C-30 para calidad de vida, ecocardiograma, prueba de esfuerzo, signos vitales y la escala FACT-Fatigue Scale. El programa de entrenamiento tuvo una duración de 36 sesiones de 70 minutos, tres veces por semana. Resultados. Al comparar los resultados post entrenamiento de las variables hemodinámicas, logramos determinar cambios en la fracción de eyección (GE1: 49,0 $\pm 5,6$ vs $52,0 \pm 5,4 ;$ GE2: $45,0 \pm 5,3$ vs $51,0 \pm 3,2 ;$ GC: $48,0 \pm 4,1$ vs 48,0 $\pm 4,4 ; p<0,005$ ), frecuencia cardiaca máxima (GE1: $155,0 \pm 7,0$ vs $159,0 \pm 4,0 ;$ GE2: $156,0 \pm 14,0$ vs $168,0 \pm$ 5,0; GC: $155,0 \pm 7,0$ vs $155,0 \pm 6,0 ; p<0,005$ ). Al igual que los resultados para calidad de vida (GE1: $55,1 \pm$ $10,3$ vs $70,9 \pm 10,2 ;$ GE2: $59,8 \pm 9,9$ vs $118,0 \pm 14,6$; GC: $60,2 \pm 7,6$ vs $62,3 \pm 9,4 ; p<0,005)$. Conclusiones. EI HIIT y MICT mejoraron significativamente la calidad de vida, fatiga, y todas las variables hemodinámicas evaluadas en la investigación. Se demostró mayores beneficios con el HIIT. El grupo control de atención habitual sin entrenamiento físico, no presentó cambios significativos.

Palabras clave: Neoplasias de la Próstata; Calidad de Vida; Rehabilitación Cardiaca; Ejercicio; Entrenamiento de Resistencia; Ensayo Clínico (fuente: DeCS BIREME).

\section{Abstract}

Introduction. Prostate cancer is the most common in men and is the second cause of death. Exercise-based rehabilitation is effective and safe in mitigating cancer effects. Objective. To determine the hemodynamic changes and quality of life in prostate cancer after a high intensity training (HIIT) or continuous to moderate intensity (MICT). Methods. A randomized clinical trial with 249 patients with stage II prostate cancer divided into 3 groups (MICT, HIIT and control group) who were applied EORTC QLQ C-30 questionnaire for quality of life, echocardiogram, stress test, vital signs and FACT-Fatigue Scale. The training program lasted 36 sessions of 70 minutes, 3 times a week. Results. When comparing the post-training results of the hemodynamic variables, we managed to determine changes in the ejection fraction (GE1: $49.0 \pm 5.6$ vs $52.0 \pm 5.4$, GE2: 45.0 \pm 5.3 vs $51.0 \pm 3.2$, GC: $48.0 \pm 4.1$ vs $48.0 \pm 4.4 p<0.005$ ), maximum heart rate (GE1: $155.0 \pm 7.0$ vs $159.0 \pm$ 4.0, GE2: $156.0 \pm 14.0$ vs $168.0 \pm 5.0, G C: 155.0 \pm 7.0$ vs $155 \pm 6.0, p<0.005)$. As in the results for quality of life (GE1: $55.1 \pm 10.3$ vs $70.9 \pm 10.2$, GE2: $59.8 \pm 9.9$ vs $118.0 \pm 14.6$, GC: $60.2 \pm 7.6$ vs $62.3 \pm 9.4, p<0.005$ ). Conclusions. HIIT and MICT significantly improved the quality of life, fatigue, and all hemodynamic variables evaluated in the research. Greater benefits were demonstrated with HIIT. The usual care control group without physical training did not show significant changes.

Keywords: Prostatic Neoplasms; Quality of Life; Cardiac Rehabilitation; Exercise; Resistance Training; Clinical Trial (source: MeSH NLM). 


\section{INTRODUCCIÓN}

El término cáncer hace referencia a un conjunto de enfermedades distinguidas por el aumento descontrolado y la multiplicación de células atípicas, Ilegando a causar la muerte. Su origen es causado por factores modificables como la alimentación, radiación, tabaco, sustancias químicas, entre otros; y los factores no modificables como problemas inmunitarios, hormonales, mutaciones heredadas y en respuesta al metabolismo ${ }^{(1)}$. El cáncer de próstata es uno de los más frecuentes y se encuentra entre las cinco causas de mortalidad en los hombres, teniendo a los adenocarcinomas en la zona periférica de la glándula, como el tipo histológico mayormente encontrado ${ }^{(2)}$.

Se conoce que el diagnóstico de cáncer causa cambios emocionales, sociales y sobre todo físicos, que originan distintas necesidades en los pacientes y sus familias. Del mismo modo, cuando mencionamos el término calidad de vida (CV) de un paciente, comprendemos esta idea como la discrepancia entre la expectativa y su realidad; en otras palabras, cuanto mayor sea la desigualdad entre los mencionados, menor será su CV (3). Asimismo, la calidad de vida relacionada con la salud (CVRS), la cual es multidimensional, predomina por la interrelación de factores individuales, sociales y ambientales ${ }^{(4)}$, y claramente diferenciable por parte del paciente de la percepción del estado de salud ${ }^{(5)}$. Participan aspectos en relación con al menos tres componentes: uno individual (relacionado con el estrés que supone la enfermedad para el paciente), uno de interacción (que tiene que ver con el apoyo social) y otro comunitario (relacionado con un sentido de pertenencia) ${ }^{(6,7)}$.

Debido a que esta situación viene seguida de un daño fisiológico y psicosocial gradual por la concentración de la enfermedad y los efectos desfavorables del tratamiento, el ejercicio físico es una de las actividades más recomendadas para el paciente. Dentro de todas las variedades de ejercicio físico disponibles, resaltamos el entrenamiento de intervalos de alta intensidad (HIIT), que esta basado en ejercicios repetidos de alta intensidad separados por cortos períodos de recu- peración. El HIIT ha sido aplicado como un esquema efectivo para la mejora de la condición física, funciones fisiológicas y factores de riesgo de enfermedad cardiovascular ${ }^{(8,9)}$. Por otro lado, otra alternativa recomendada es el ejercicio aeróbico a intensidad moderada continúa (MICT), que ha demostrado ser seguro y eficiente en pacientes oncológicos ${ }^{(10)}$. Estudios recientes muestran que el ejercicio a intensidades más altas puede ofrecer diferentes y adicionales beneficios para las personas con cáncer ${ }^{(11)}$. Resaltando que diferentes estudios han evidenciado que cuando el paciente realiza ejercicio con intensidad moderada (tres horas a la semana) presenta una menor tasa de recaída por el cáncer (57\%) y un menor riesgo de mortalidad (Aproximadamente, $30 \%)$.

Así, el objetivo de la presente investigación fue determinar los cambios hemodinámicos y calidad de vida en pacientes con cáncer de próstata (estadio II) luego de un programa de entrenamiento HIIT, en comparación con un programa MICT.

\section{MÉTODOS}

\section{Diseño del estudio}

Ensayo clínico aleatorizado, que se realizó con una muestra de 249 pacientes con cáncer en estadio II, cuya duración fue de 3 años y 4 meses (noviembre 2015- febrero 2019), en el Centro de Rehabilitación Rehabilitar Cúcuta, Colombia. El presente artículo se da como resultado del macroproyecto randomizado de ONCO-EXE TRIAL registrado en el sistema de registro y resultados de protocolo ClinicalTrials.gov de la National Library of Medicine (NLM), the National Institutes of Health (NIH) y la Food and Drug Administration (FDA): NCT03915288.

\section{Participantes}

Los pacientes tenían características similares en relación a: etapa del cáncer, fracción de eyección, clase funcional, porcentaje muscular, grasa e IMC (índice de masa corporal), circunferencia abdominal, sobrepeso, obesidad, prevalencia de diabetes, hipertensión arterial (HTA), factores de riesgo cardiovascular. Además, todos los pacientes mostraron "riesgo alto" según la estratificación pro- puesta por la Asociación Americana de Rehabilitación Cardiopulmonar (12).

Para la inclusión en el estudio, los pacientes tenían que estar diagnosticados con cáncer en estadío II, tener más de 18 años y asistir a un programa de entrenamiento. Asimismo, todos los pacientes de forma personal firmaron un consentimiento informado protegido por el Comité de Ética e Investigación de la Institución Prestadora de Servicios Rehabilitar Cúcuta, Colombia. De la misma manera, fue obligatorio que los individuos tuvieran una fracción de eyección superior al $35 \%$, sin dificultad para realizar los cuestionarios, pruebas, y las medidas que requiere la investigación; como también, el compromiso de asistir tres veces por semana para entrenamiento.

Se excluyeron los pacientes con dolor intenso en miembros inferiores, angina inestable, frecuencia cardíaca >120 lpm (latidos por minuto) en reposo, presión arterial sistólica $>190 \mathrm{mmHg}$, presión arterial diastólica $>120 \mathrm{mmHg}$. También, se excluyeron pacientes con cáncer en otro estadío que no fuera el II. De la misma manera, determinamos que el individuo tenía la elección de abandonar la investigación cuando lo quisiera o mostrar inestabilidad hemodinámica sin mejoría durante ninguna prueba o durante el proceso de intervención.

\section{Intervenciones}

Inicialmente, el paciente fue evaluado en el área de oncología para conocer su estado actual, las particularidades sociodemográficas, antropométricas y fisiológicas. El mismo día, fue evaluado por un fisioterapeuta para realizar una prueba de esfuerzo de acuerdo con el protocolo de Naughton, que se recomienda en pacientes de alto riesgo y cuya velocidad e inclinación en la banda sin fin es por fases de dos minutos, necesaria para determinar la frecuencia cardíaca máxima (FCM) y con fundamento a esta, prescribir el ejercicio conveniente. Para estos estudios, el paciente tenía prohibido fumar, beber o ingerir algún medicamento que pudiera alterar sus signos vitales o el rendimiento en la prueba.

Para la intervención de los pacientes se trabajó según la FCM obtenida en la prueba de esfuerzo con el protocolo de 
Naughton que realizaron los pacientes el primer día de ingreso. Para la fuerza, se utilizó el resultado del test de 1RM. El programa de entrenamiento tuvo una duración de 36 semanas con asistencia de tres veces a la semana con 70 minutos por intervención. Durante toda la intervención el paciente estuvo monitorizado por un sistema Polar Multisport RS800CX ${ }^{\circledR}$, oximetría y con la escala de Borg para evitar sobrepasar la intensidad de entrenamiento. La inclinación, resistencia, y velocidad de los ejercicios fueron asignadas según los parámetros indicativos (FCM, $\mathrm{VO}_{2}$, Borg) para intensidad moderada y alta.

En el grupo experimental 1 (GE 1), el programa de intervención consistió en 70 minutos por sesión, en donde $10 \mathrm{mi}$ nutos eran de calentamiento (ejercicios respiratorios, caminata, estiramientos), 30 minutos de entrenamiento aeróbico continuo a intensidad moderada (60$80 \%$ FCM) en bicicleta, remo, elíptica y recumbent. Los otros 20 minutos, fueron para el entrenamiento de fuerza (40-60\% fuerza máxima) con mancuernas y theraband de manera progresiva. Y los últimos 10 minutos, fueron para el enfriamiento (ejercicios de coordinación, equilibrio, caminata y ejercicios respiratorios).

Mientras, en el grupo experimental 2 (GE 2), los 10 minutos de calentamiento fueron similares al GE1. Los 30 minutos de entrenamiento fueron de ejercicio interválico HIIT. Es decir, los pacientes fueron intervenidos durante los 30 minutos con un protocolo creado por el autor principal, denominado 30-30: 30 segundos a intensidad moderada (60-80\% FCM) y 30 segundos a intensidad alta (80-90\% FCM) en banda sin fin, bicicleta estática, remo y recumbent. El entrenamiento de fuerza y enfriamiento fue idéntico al GE1.

Finalmente, se consideró la conformación de un grupo control (GC), en pacientes que recibirían el cuidado usual e institucionalizado que se da en estos casos, sin la adición de ningún esquema de ejercicio físico.

\section{Variables de respuesta}

\section{Características antropométricas}

De manera global, se obtuvieron los siguientes datos de los participantes: ante- cedentes familiares y personales usando un cuestionario de ejecución individual; y medidas antropométricas (peso, talla, índice de masa corporal, circunferencia abdominal, porcentaje de grasa y músculo) llevando a cabo procedimientos estandarizados en la población colombiana.

El peso, el porcentaje de grasa y el músculo se consiguieron por medio de la balanza digital Tezzio TB-30037 ${ }^{\circledR}$ calibrada con antelación y colocada en una superficie plana y estable, efectuando las instrucciones del manual del usuario. Por otro lado, la talla se alcanzó con el Adult Acrylic Halter Wall Kramer $2104{ }^{\circledR}$, colocando al paciente en bipedestación, con la cabeza en el plano de Frankfort y los hombros relajados para prevenir la lordosis y los miembros inferiores totalmente contra la pared. Finalmente, con estas variables se estableció el IMC. Después, con una cinta métrica y una exactitud de $1 \mathrm{~mm}$, se tomó la medida de la circunferencia abdominal tomando la referencia anatómica descrita por Frisancho ${ }^{(13)}$.

\section{Parámetros clínicos y hemodinámicos}

De manera global los pacientes fueron sometidos a un ecocardiograma 2-D (bidimensional) realizado por un médico cardiólogo externo al equipo de investigación y quien generó una valoración antes y después al protocolo de ejercicios para determinar la conformación, fracción de eyección del ventrículo izquierdo (FEVI) e investigar su movimiento en el momento. Dentro de la evaluación, se estableció la clase funcional de cada individuo conforme a la clasificación de la New York Heart Assosiation, que denomina 4 clases (I, II, III y IV) basadas en las limitaciones de la actividad física del paciente, causados por síntomas cardíacos. Del mismo modo, la disnea percibida y el esfuerzo se evaluaron utilizando la escala de Borg modificada ${ }^{(14)}$. La frecuencia cardíaca fue valorada por el sistema Polar Multisport RS800CX ${ }^{\circledR}$ y la respiratoria, al igual que la presión arterial sistólica y diastólica, se consiguieron manualmente y corroborada por un segundo evaluador. En tanto que la saturación de oxígeno se consiguió con un oxímetro portátil Nellcor Puritan Bennett ${ }^{\circledR}$.

\section{Calidad de vida}

Para este apartado, se utilizó el cuestionario EORTC QLQ C-30 (European Organisation for Research and Treatment of Cancer quality of Life Questionnaire Core 30$){ }^{(15)}$ con su respectiva interpretación siguiendo la guía del manual de la European Organisation for Research and Treatment of Cancer ${ }^{(16)}$. Esta prueba fue solicitada en el idioma español a los autores principales EORTC Quality of Life Group website quienes aprobaron su uso en la presente investigación. La interpretación de un resultado alto representó una "alta / saludable" calidad de vida del participante evaluado y tomando como valor de referencia 65 puntos como indicativo de una baja calidad de vida.

\section{Fatiga}

La fatiga se valoró mediante la escala FACT-Fatigue Scale (Escala de Fatiga de la Evaluación Funcional de la Terapia del Cáncer), que es un registro de 13 ítems que valora la gravedad de la fatiga asociada al cáncer (FAC) en la última semana, con una escala de 0 a 4 para un total de 52 puntos, y que con puntuaciones más altas reflejan una FAC más alta (mayor fatiga). Resaltando que resultados inferiores a 26 se considera una FAC baja (menor fatiga) ${ }^{(21)}$.

\section{Fuerza}

La fuerza fue valorada mediante dinamometría con el Hand Grip CAMRY Electronic hand dynamometer model EH1O1 ${ }^{\circledR}$. Para este test se solicitó cumplir con la siguiente técnica: sujeto de pie o sentado en posición cómoda y sin apoyabrazos. Hombros aducidos y sin rotación. Codo flexionado a 90. Antebrazo y muñeca en posición neutra. Se evaluó la fuerza de prensión en la mano hábil y se consignaron tres determinaciones seguidas, respetando el tiempo de recuperación muscular, que fue de alrededor un minuto. Por otra parte, para la determinación del peso inicial del entrenamiento de fuerza para las extremidades superiores (MMSS) e inferiores (MMII), se aplicó el test de una repetición máxima (1RM) con una extensión completa del grupo muscular utilizado, sin sustituciones musculares. 


\section{Aleatorización}

La distribución de los pacientes seleccionados a los grupos experimentales se realizó mediante un muestro probabilístico básico por medio de una tabla de números aleatorios, cuyo orden fue randomizado mediante el programa Microsoft Excel $2016{ }^{\circledR}$.

\section{Enmascaramiento}

Los pacientes fueron evaluados inicialmente por un profesional no investigador (médico oncólogo del servicio de oncología). Posteriormente, fueron registrados en una base de datos en Microsoft Excel $2016^{\circledR}$, únicamente con un número de identificación que permitió el cegamiento de los autores. Todos los individuos y profesionales incluyendo a los investigadores fueron cegados a lo largo de todo el protocolo.

Los investigadores realizaron los formularios y las pruebas sin el conocimiento de ellos a la asignación de cada paciente, tipo de cáncer, o afectación del participante. Los investigadores encargados de la realización de las pruebas respectivas fueron cegados durante todo el proceso (D. P-F. y P. P-R.). Después de las pruebas, se sugirió a los participantes que se acercaran al autor principal (J. P-R) para comunicar su horario y la fecha de inicio del programa de rehabilitación. Del mismo modo, destacamos que, desde el comienzo de los exámenes hasta el final del programa de entrenamiento, los autores no establecieron una conversación sobre el tema con los participantes o los terapeutas. Únicamente, los autores efectuaron los cuestionarios, test y pruebas pre y post entrenamiento. $Y$ solo el autor J. P-R. sustentó reuniones periódicas con los fisioterapeutas entrenadores para saber y acordar el entrenamiento de los pacientes, pero no para interactuar con los participantes o revisar sus datos.

Luego del programa de entrenamiento, se llevaron a cabo pruebas y exámenes a los pacientes para cuantificar las modificaciones luego del entrenamiento. Teniendo presente la información recopilada antes y después, los análisis estadísticos se efectuaron de modo cegado por los autores R. P-R. y K. S-P. Por último, una vez que las diferentes variables se estudiaron a ciegas, todos los autores fueron notificados para las conclusiones respectivas.

\section{Análisis estadístico}

Se realizó una base de datos en Microsoft Excel $2016{ }^{\circledR}$ con todos los individuos y sus resultados de las pruebas, test y cuestionarios pre y post entrenamiento. Luego, se efectuaron las estadísticas descriptivas para evaluar y señalar los datos por promedios con su desviación estándar correspondiente. La normalidad de los datos se evaluó mediante la prueba de Kolmogorov-Smirnov y la indicación de especificidad fue evidente para todos los análisis. Asimismo, a través de la prueba de Tukey, se utilizó el análisis de varianza ANOVA (análisis de varianza de una vía) y, posteriormente, las pruebas post hoc para evaluar las características de los diferentes grupos de edad, género y antropometría. En todos los casos,

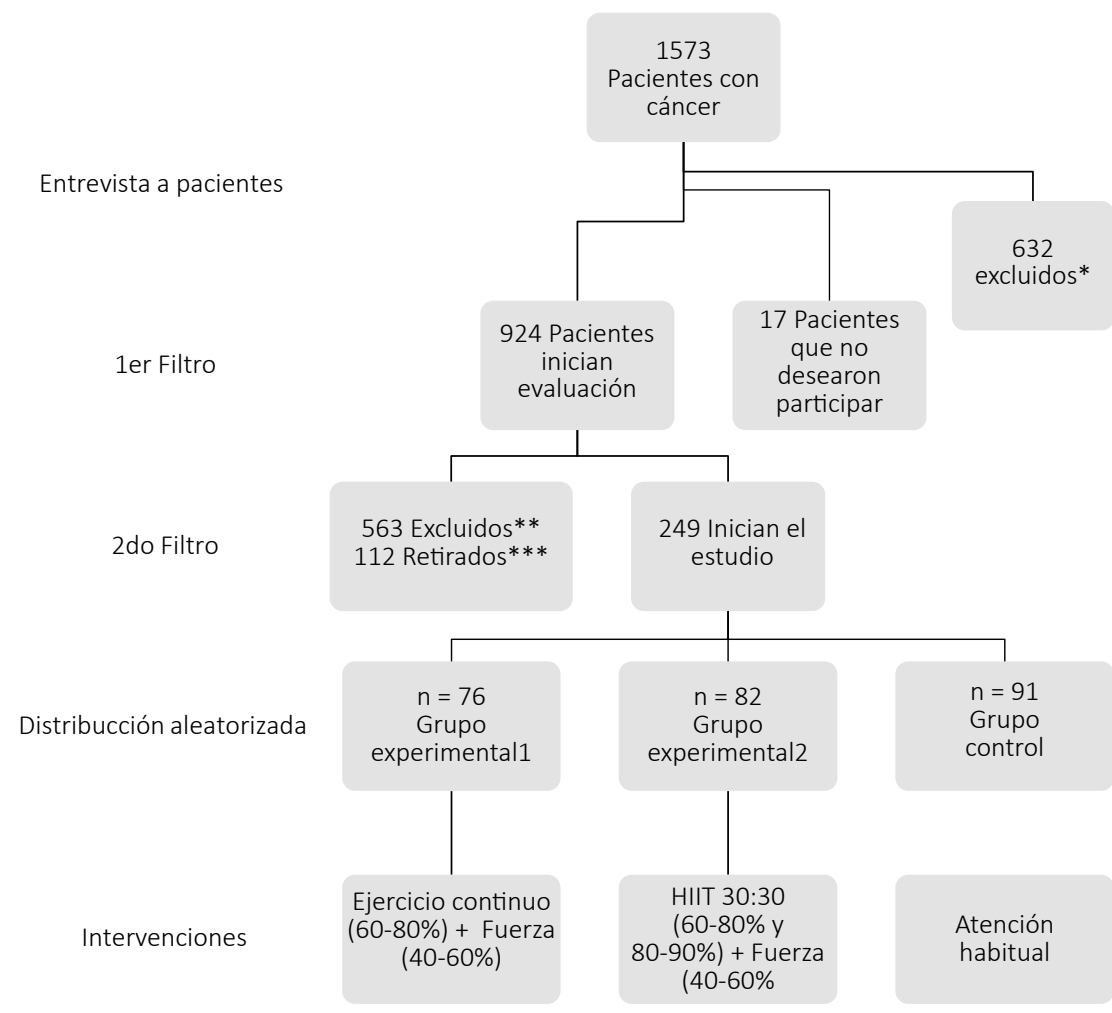

HIIT: High Intensity Interval Training

*632 Pacientes excluidos inicialmente (132 por cáncer fase 1; 351 por cáncer fase 3; 149 por cáncer fase 4). **563 Pacientes excluidos por no cumplir criterios de inclusión (otros tipos de cáncer: 216 cáncer de mama, 79 colorrectal, 70 tiroides, 48 cervico-uterino, 37 pulmón, 29 estomago, 25 hepático, 24 páncreas, 22 riñón, 13 esófago). ***112 Pacientes excluidos por contraindicación ( 5 por proceso infeccioso, 13 dificultad para el traslado al sitio de entrenamiento, 22 por alteraciones arrítmicas, 35 por hipertensión sistólica $>190 \mathrm{mmHg}, 37$ dolor articular).

Figura 1. Flujograma de la distribución de los grupos $(n=249)$ para la evaluación experimental de cambios hemodinámicos y calidad de vida en pacientes con cáncer de próstata luego de un entrenamiento HIIT y MICT. 
En cuanto al nivel académico de los participantes, el 46,2\% (n=120) había completado solo la escuela primaria, $13,9 \%(n=25)$ estudió hasta la educación secundaria, $17,1 \%(n=45)$ nivel universitario y el $22,6 \%(n=59)$ no estudió. Los tratamientos oncológicos realizados fueron: cirugía (24\%), radioterapia (65\%), quimioterapia (55\%), terapia hormonal (37\%). Respecto a los factores de riesgo más frecuentes en la población de estudio fueron: sedentarismo, obesidad abdominal, sobrepeso y obesidad, hipertensión arterial, tabaquismo, dislipidemia, dieta inadecuada, diabetes mellitus, entre otros (Tabla 1).

Al comparar los resultados post-entrenamiento de las variables hemodinámi- cas, logramos determinar cambios en la fracción de eyección (GE 1: 49,0 $\pm 5,6$ vs $52,0 \pm 5,4 ;$ GE $2: 45,0 \pm 5,3$ vs $51,0 \pm 3,2$; GC: $48,0 \pm 4,1$ vs $48,0 \pm 4,4 ; p<0,005)$, frecuencia cardíaca máxima (GE 1: 155,0 $\pm 7,0$ vs $159,0 \pm 4,0 ;$ GE $2: 156,0 \pm 14,0$ vs $168,0 \pm 5,0 ;$ GC: $155,0 \pm 7,0$ vs 155,0 $\pm 6,0 ; p<0,005)$, presión arterial sistólica (GE 1: $145,0 \pm 10,6$ vs $140,0 \pm 4,4$; GE 2: $130,0 \pm 8,3$ vs $121,0 \pm 1,2 ;$ GC: $140,0 \pm 7,2$ vs $140,0 \pm 6,6 ; p<0,005)$ y diastólica (GE 1: $95,0 \pm 6,7$ vs $90,0 \pm 3,1 ;$ GE $2: 85,0 \pm$ 11,0 vs $80,0 \pm 2,2 ;$ GC: $90,0 \pm 4,5$ vs 90,0 $\pm 5,7 ; p<0,005)$.

En forma similar, se encontraron diferencias estadísticamente significativas en los resultados del cuestionario EORTC QLQ C-30 para calidad de vida (GE 1: 55,1 $\pm 10,3$ vs $70,9 \pm 10,2 ;$ GE $2: 59,8 \pm 9,9$ vs $118,0 \pm 14,6$; GC: $60,2 \pm 7,6$ vs $62,3 \pm 9,4$; $p<0,005)$. Además, un factor influyente en la calidad de vida es la fatiga asociada al cáncer de próstata; para la cual, también se obtuvieron cambios luego de un programa de entrenamiento MICT $(22,8$ $\pm 4,8$ vs $15,7 \pm 7,9 ; p<0,005)$ y HIIT $(25,3 \pm$ 7,4 vs $9,1 \pm 8,1 ; p<0,005$ ) (Figura 2).

Desde el punto de vista antropométrico, al revisar el porcentaje de músculo, grasa e IMC antes y después de la intervención de cada programa de entrenamiento basados en el MICT (Grupo 1) y HIIT (Grupo 2), con un componente de fuerza muscular, se observó una mejora significativa en todas las variables $(p<0,05)$. Además, al comparar los re-

Tabla 1. Características iniciales de la población de estudio $(n=249)$. Pacientes con cáncer de próstata luego de un entrenamiento HIIT y MICT.

\begin{tabular}{|c|c|c|c|}
\hline Características & $\begin{array}{l}\text { Grupo experimental } 1 \\
\qquad n=76\end{array}$ & $\begin{array}{l}\text { Grupo experimental } 2 \\
\qquad n=82\end{array}$ & $\begin{array}{l}\text { Grupo control } \\
\quad n=91\end{array}$ \\
\hline Edad (años) & $55,0 \pm 3,0$ & $54,0 \pm 3,0$ & $52,0 \pm 1,0$ \\
\hline Fracción de eyección (\%) & $49,0 \pm 5,6$ & $45,0 \pm 5,3$ & $48,0 \pm 4,1$ \\
\hline FCM en prueba de esfuerzo & $155,0 \pm 7,0$ & $156,0 \pm 14,0$ & $158,0 \pm 11,0$ \\
\hline PAS (mmHg) & $145,0 \pm 10,6$ & $130,0 \pm 8,3$ & $140,0 \pm 7,2$ \\
\hline PAD (mmHg) & $95,0 \pm 6,7$ & $85,0 \pm 11,0$ & $90,0 \pm 4,5$ \\
\hline Altura (m) & $1,6 \pm 10,2$ & $1,6 \pm 4,9$ & $1,6 \pm 3,4$ \\
\hline Peso (Kg) & $75,0 \pm 11,1$ & $82,0 \pm 9,6$ & $80,0 \pm 7,3$ \\
\hline IMC & $28,2 \pm 5,4$ & $32,8 \pm 3,9$ & $32,4 \pm 4,2$ \\
\hline Circunferencia abdominal (cm) & $88,0 \pm 3,2$ & $92,0 \pm 5,5$ & $91,0 \pm 4,5$ \\
\hline Porcentaje graso (\%) & $21,0 \pm 4,7$ & $27,0 \pm 6,2$ & $25,0 \pm 7,6$ \\
\hline Porcentaje muscular (\%) & $31,0 \pm 11,4$ & $30,0 \pm 9,6$ & $30,0 \pm 11,8$ \\
\hline Fuerza & $24,5 \pm 10,5$ & $27,4 \pm 5,7$ & $29,7 \pm 6,3$ \\
\hline Fatiga (FACT-F) & $22,8 \pm 4,8$ & $25,3 \pm 7,4$ & $21,6 \pm 3,2$ \\
\hline Calidad de vida & $55,1 \pm 10,3$ & $59,8 \pm 9,9$ & $60,2 \pm 7,6$ \\
\hline Sobrepeso y/u obesidad & $63 \%$ & $75 \%$ & $69 \%$ \\
\hline Obesidad abdominal & $70 \%$ & $76 \%$ & $72 \%$ \\
\hline Dislipidemia & $41 \%$ & $37 \%$ & $23 \%$ \\
\hline Hipertensión arterial & $55 \%$ & $31 \%$ & $46 \%$ \\
\hline Diabetes & $23 \%$ & $24 \%$ & $18 \%$ \\
\hline Enfermedad renal & $4 \%$ & $3 \%$ & $5 \%$ \\
\hline Sedentarismo* & $92 \%$ & $94 \%$ & $91 \%$ \\
\hline Tabaquismo & $61 \%$ & $57 \%$ & $43 \%$ \\
\hline Alcoholismo & $5 \%$ & $7 \%$ & $3 \%$ \\
\hline Ingesta de comida inadecuada & $31 \%$ & $23 \%$ & $19 \%$ \\
\hline Historial de IAM & $3 \%$ & $2 \%$ & $4 \%$ \\
\hline
\end{tabular}

FCM: frecuencia cardíaca máxima; IAM: infarto agudo de miocardio.

* Menos de 150 minutos por semana. 


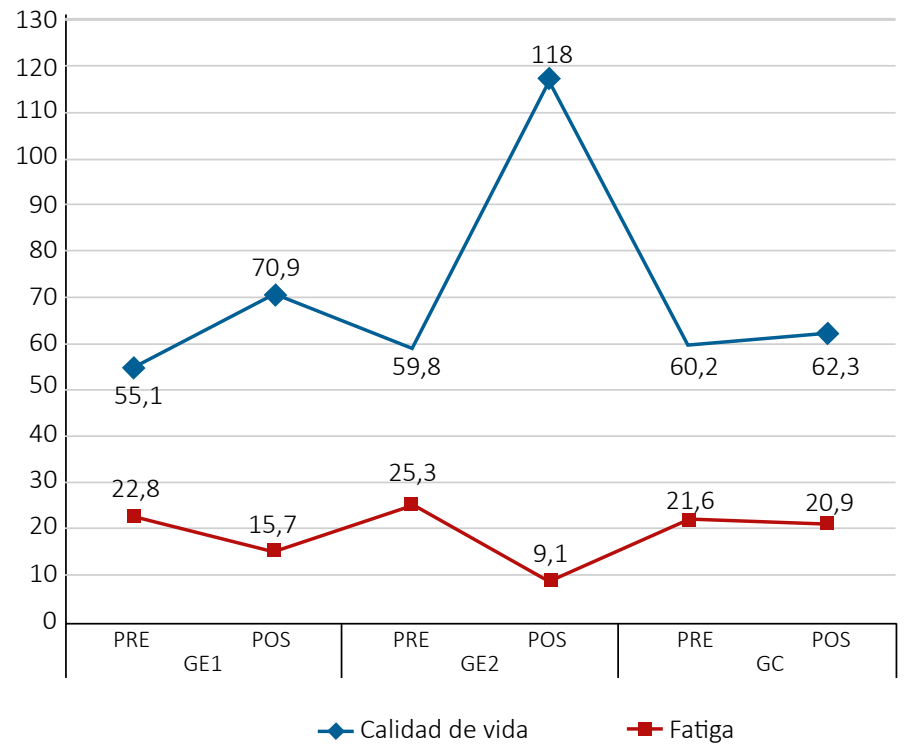

Figura 2. Calidad de vida y fatiga en pacientes con cáncer de próstata luego de un entrenamiento HIIT Y MICT. Para la determninación de una calidad de vida alta o saludable, puntaje mayor a 65. Para la determinación de menor fatiga asociada a cáncer, puntaje menor a 26. Los puntajes presentados corresponden a los promedios por cada grupo. GE1: grupo experimental 1. GE2: grupo experimental 2. GC: grupo control.

sultados entre los grupos, fue posible demostrar mejores resultados con una diferencia significativa en el grupo experimental $2(p<0,05)$. Para el grupo control no hubo mejoría en la mayoría de variables evaluadas (Tabla 2).

Durante los programas de intervención no se presentaron complicaciones ni eventos adversos debido al entrenamiento físico realizado por los pacientes con cáncer de próstata en estadio II. Además, durante las sesiones de los programas de entrenamiento, los participantes informaron una disnea percibida según la escala de Borg entre 6 y 8 (intensidad moderada) en los grupos 1 y 2 , resaltando que el grupo 2 tuvo picos entre 9 y 10, así como la sensación de fatiga durante el ejercicio.

\section{DISCUSIÓN}

En los hallazgos de esta investigación resaltan el impacto e importancia de un programa de entrenamiento físico inspeccionado y estructurado sobre la fatiga y los distintos aspectos de la calidad de vida: física, funcional, emocional y bienestar social.

Latham y col. ${ }^{(18)}$ y Bernat-Carles Serdà (19), coindicen en que la fuerza transfiere tanto la capacidad de ejecución técnica como en la calidad de los patrones motores básicos. La mejora de la fuerza en las extremidades inferiores reduce el riesgo de la triada caída, fractura y dependencia, descrita en el

Tabla 2. Cambios post-entrenamiento HIIT y MICT en pacientes con cáncer de próstata $(n=249)$.

\begin{tabular}{|c|c|c|c|c|c|c|c|c|c|}
\hline \multirow[t]{2}{*}{ Variables } & \multicolumn{2}{|c|}{$\begin{array}{l}\text { Grupo experimental } 1 \\
\qquad n=76\end{array}$} & \multicolumn{2}{|c|}{$\begin{array}{l}\text { Grupo experimental } 2 \\
\qquad \mathbf{n = 8 2}\end{array}$} & \multicolumn{2}{|c|}{$\begin{array}{l}\text { Grupo control } \\
\quad n=91\end{array}$} & \multirow{2}{*}{$\begin{array}{c}\text { GE } 1 \\
\text { Vs } \\
\text { GE } 2 \\
\text { Valor } p\end{array}$} & \multirow{2}{*}{$\begin{array}{c}\text { GE } 1 \\
\text { Vs } \\
\text { GC } \\
\text { Valor } \\
\mathbf{p}\end{array}$} & \multirow{2}{*}{$\begin{array}{c}\text { GE } 2 \\
\text { Vs } \\
\text { GC } \\
\text { Valor } \\
\mathbf{p}\end{array}$} \\
\hline & Pre & Post & Pre & Post & Pre & Post & & & \\
\hline Fracción de eyección (\%) & $49,0 \pm 5,6$ & $52,0 \pm 5,4$ & $45,0 \pm 5,3$ & $51,0 \pm 3,2$ & $48,0 \pm 4,1$ & $48,0 \pm 4,4$ & 0,003 & 0,000 & 0,000 \\
\hline FCM (Ipm) & $155,0 \pm 7,0$ & $159,0 \pm 4,0$ & $156,0 \pm 14,0$ & $168,0 \pm 5,0$ & $155,0 \pm 7,0$ & $155,0 \pm 6,0$ & 0,012 & 0,000 & 0,001 \\
\hline PAS (mmHg) & $145,0 \pm 10,6$ & $140,0 \pm 4,4$ & $130,0 \pm 8,3$ & $121,0 \pm 1,2$ & $140,0 \pm 7,2$ & $140,0 \pm 6,6$ & 0,001 & 0,002 & 0,006 \\
\hline PAD (mmHg) & $95,0 \pm 6,7$ & $90,0 \pm 3,1$ & $85,0 \pm 11,0$ & $80,0 \pm 2,2$ & $90,0 \pm 4,5$ & $90,0 \pm 5,7$ & 0,000 & 0,000 & 0,001 \\
\hline Peso (Kg) & $75,0 \pm 11,1$ & $71,0 \pm 6,2$ & $82,0 \pm 9,6$ & $70,0 \pm 3,8$ & $80,0 \pm 7,3$ & $81,0 \pm 4,4$ & 0,001 & 0,000 & 0,000 \\
\hline Índice de masa corporal & $28,2 \pm 5,4$ & $26,7 \pm 2,8$ & $32,8 \pm 3,9$ & $28,0 \pm 1,3$ & $32,4 \pm 4,2$ & $32,8 \pm 7,1$ & 0,001 & 0,002 & 0,001 \\
\hline $\mathrm{CA}(\mathrm{cm})$ & $88,0 \pm 3,2$ & $82,0 \pm 8,0$ & $92,0 \pm 5,5$ & $80,0 \pm 1,0$ & $91,0 \pm 4,5$ & $91,0 \pm 4,9$ & 0,000 & 0,001 & 0,000 \\
\hline Porcentaje graso (\%) & $21,0 \pm 4,7$ & $17,0 \pm 3,3$ & $27,0 \pm 6,2$ & $19,0 \pm 2,4$ & $25,0 \pm 7,6$ & $26,0 \pm 6,2$ & 0,001 & 0,002 & 0,001 \\
\hline $\begin{array}{l}\text { Porcentaje muscular } \\
(\%)\end{array}$ & $31,0 \pm 11,4$ & $32,0 \pm 3,5$ & $30,0 \pm 9,6$ & $38,0 \pm 6,7$ & $30,0 \pm 11,8$ & $31,0 \pm 8,4$ & 0,000 & 0,001 & 0,001 \\
\hline Fuerza (Kg) & $24,5 \pm 10,5$ & $29,9 \pm 7,8$ & $27,4 \pm 5,7$ & $38,2 \pm 3,1$ & $29,7 \pm 6,3$ & $29,5 \pm 4,8$ & 0,001 & 0,002 & 0,001 \\
\hline Fatiga (FACT-F)* & $22,8 \pm 4,8$ & $15,7 \pm 7,9$ & $25,3 \pm 7,4$ & $9,1 \pm 8,1$ & $21,6 \pm 3,2$ & $20,9 \pm 8,9$ & 0,012 & 0,006 & 0,000 \\
\hline Calidad de vida* & $55,1 \pm 10,3$ & $70,9 \pm 10,2$ & $59,8 \pm 9,9$ & $118,0 \pm 14,6$ & $60,2 \pm 7,6$ & $62,3 \pm 9,4$ & 0,005 & $<0,001$ & 0,002 \\
\hline
\end{tabular}

FCM: frecuencia cardíaca máxima; Lpm: latidos por minuto; CA: Circunferencia abdominal.

Los puntajes presentados corresponden a los promedios por cada grupo. Fatiga de 0 - 52 y Calidad de vida de 0 - 126 . Para la determninación de una calidad de vida alta o saludable, puntaje mayor a 65. Para la determinación de menor fatiga asociada a cáncer, puntaje menor a 26.

GE1: grupo experimental 1. GE2: grupo experimental 2. GC: grupo control. 
síndrome geriátrico. Por otro lado, Segal RJ y col. ${ }^{(20)}$ concluyeron que el ejercicio de resistencia reduce la fatiga y mejora la calidad de vida así como la idoneidad muscular en hombres con cáncer de próstata que reciben terapia de privación de andrógenos, y que este tipo de ejercicio puede ser un componente importante de la atención de apoyo para estos pacientes.

Craike MJ y col. (21), reportaron que luego de los programas de HIIT y MICT los pacientes mejoraron significativamente la presión arterial sistólica y los dominios de tiempo de la variabilidad de la frecuencia cardiaca, incluido el número de intervalos e intervalos entre latidos; sin embargo, la relación InLF / alta frecuencia sólo mejoró significativamente en el grupo HIIT después de ocho sesiones de entrenamiento. Liou G. y col. ${ }^{(22)}$ resaltaron el fenómeno que potencia el crecimiento del tumor y regula al alza la inflamación: la sobreexpresión de especies de oxígeno reactivas (ROS) causado por reacciones inflamatorias persistentes. El HIIT parece moderar esta vía en comparación con MICT mediante el aumento del glutatión peroxidasa (GPx), una enzima con efectos protectores contra ROS y potencial terapéutica para enfermedades malignas.

En cuanto a la calidad de vida, según la evidencia científica, los pacientes con prostatectomía radical laparoscópica (PRL) tienen mayor deterioro debido a las consecuencias de la cirugía o la necesidad de terapias adyuvantes y su toxicidad (radioterapia y hormonoterapia). Asimismo, el mayor impacto se da en pacientes que presentan incontinencia urinaria posquirúrgica y/o síntomas urinarios de llenado o de vaciamiento severos, asociados a fibrosis de la anastomosis uretrovesical (23). Bill A. y col. ${ }^{(24)}$ refirieron que los pacientes de mayor edad justifican sus síntomas utilizando mecanismos compensatorios de la edad, por ejemplo, en la función eréctil; y en el caso de la incontinencia urinaria existe la creencia que es la consecuencia por librarse de la enfermedad. Asimismo, Veiga FJ. y col. (25) manifiestaron que los pacientes más jóvenes se preocupan de forma importante por la función eréctil, y esto genera mayor ansiedad e impacto en la calidad de vida. Algunos se perciben como mutilados, a pesar de haber sido informados acerca de las consecuencias de la cirugía.
Las principales limitaciones de nuestro estudio estan relacionadas a la duración de la intervención; así, aunque se encontró un efecto positivo en las variables evaluadas, estas podrían variar de un paciente a otro según el tiempo de evolución de la enfermedad del paciente y el tratamiento correspondiente. Por otro lado, el control de variables asociadas a la alimentación y el control de peso entre los grupos experimentales podría determinar la variación de los resultados.

Finalmente, se concluye que el entrenamiento físico se mostró seguro y beneficioso para pacientes con cáncer de próstata en estadio II. Resaltando que el entrenamiento HIIT y MICT mejoraron significativamente la calidad de vida, fatiga asociada al cáncer de próstata y todas las variables hemodinámicas evaluadas en esta investigación. Así, se demostró mayores beneficios en los pacientes que recibieron HIIT en comparación con el grupo MICT y el grupo control. Asimismo, el grupo control de atención habitual sin entrenamiento físico ni ejercicio supervisado, no presentó cambios significativos ni mejoras en la calidad de vida, fatiga asociada al cáncer ni variables hemodinámicas (FEVI, FCM, PAS, PAD).

\section{REFERENCIAS BIBLIOGRÁFICAS}

1. American Cancer Society. Datos y estadísticas sobre el cáncer entre los hispanos/latinos 2012-2014. Atlanta: American Cancer Society; 2012.

2. Delgado D. Cáncer de próstata: etiologia diagnóstico y tratamiento. Rev Med Cos Cen 2016;73(620):707-10.

3. Castañeda de la Lanza C, O'Shea GJ, Narváez M, Lozano J, Castañeda G, Castañeda J. Calidad de vida y control de sintomas en el paciente oncológico. GAMO. 2015;14(3):150-6. DOI: 10.1016/j. gamo.2015.07.007

4. Skevington SM, Dehner S, Gillison FB, McGrath EJ, Lovell CR. How appropriate is the WHOQOLBREF for assessing the quality of life of adolescents? Psychol Health. 2014;29(3):297-317. DOI 10.1080/08870446.2013.845668

5. Anota A, Bascoul-Mollevi C, Conroy T, Guillemin F, Velten $\mathrm{M}$, Jolly $\mathrm{D}$, et al. Item response theory and factor analysis as a mean to characterize occurrence of response shift in a longitudinal quality of life study in breast cancer patients. Health Qual Life Outcomes. 2014;12:32. DOI: 10.1186/1477-7525-12-32

6. Sánchez Pedraza R, Sierra Matamoros FA, Martín Cardinal E. ¿Qué es calidad de vida para un paciente con cáncer? Avances en Psicología Latinoamericana. 2015;33(3):371-85. DOI: 10.12804/ revistas.urosario.edu.co/apl/a.3215
7. Diz R, Garza A, Olivas E, Montes J, Fernández G. Cáncer y depresión: una revisión. Psicología y Salud. 2019;29(1):115-24. DOI: 10.25009/pys. v29i1.2573

8. Petersen B, Hastings B, Gottschall J. High Intensity Interval Cycling Improves Physical Fitness in Trained Adults. Journal of Fitness Research. 2016;5(1):39-47.

9. Ramírez-Vélez R, Tordecilla-Sanders A, Téllez-T LA, Camelo-Prieto D, Hernández-Quiñonez PA, Correa-Bautista JE, et al. Effect of Moderate Versus High-Intensity Interval Exercise Training on Heart Rate Variability Parameters in Inactive Latin-American Adults: A Randomised Clinical Trial. J Strength Cond Res. 2017; Feb 1. DOI: 10.1519/ JSC.0000000000001833

10. Devin JL, Sax AT, Hughes GI, Jenkins DG, Aitken JF, Chambers SK, et al. The influence of high-intensity compared with moderate-intensity exercise training on cardiorespiratory fitness and body composition in colorectal cancer survivors: a randomised controlled trial. J Cancer Surviv. 2016;10(3):467-79. DOI: 10.1007/s11764-015-0490-7

11. Toohey K, Pumpa KL, Arnolda L, Cooke J, Yip D, Craft PS, et al. A pilot study examining the effects of low-volume high-intensity interval training and continuous low to moderate intensity training on quality of life, functional capacity and cardiovascular risk factors in cancer survivors. PeerJ. 2016;4:e2613. DOI: 10.7717/peerj.2613

12. Thomas RJ, Balady G, Banka G, Beckie TM, Chiu J, Gokak S, et al. 2018 ACC/AHA Clinical Performance and Quality Measures for Cardiac Rehabilitation: A Report of the American College of Cardiology/American Heart Association Task Force on Performance Measures. J Am Coll Cardiol. 2018;71(16):1814-37. DOI: 10.1016/j.jacc.2018.01.004

13. Frisancho R. Anthropometric Standards for the Assessment of Growth and Nutritional Status [On line]. Michigan: The University of Michigan Press; 1990 [citado 15 enero 2019]. Disponible en: https:// www.press.umich.edu/12198/anthropometric_standards_for_the_assessment_of_growth_and_nutritional_status

14. Fett CA, Fett WCR, Marchini JS. Exercício resistido vs jogging em fatores de risco metabólicos de mulheres com sobrepeso/obesas. Arquivos Brasileiros de Cardiologia. 2009;93(5):519-25. DOI: 10.1590/ S0066-782X2009001100013

15. Aaronson NK, Ahmedzai S, Bergman B, Bullinger M, Cull A, Duez NJ, et al. The European Organization for Research and Treatment of Cancer QLQ-C30: a quality-of-life instrument for use in international clinical trials in oncology. J Natl Cancer Inst. 1993;85(5):365-76. DOI: 10.1093/jnci/85.5.365

16. Fayers P, Aaronson NK, Bjordal K, Groenvold M, Curran D, Bottomley A. EORTC QLQ-C30 Scoring Manual [On line]. European Organisation for Research and Treatment of Cancer; 2001 [citado 15 enero 2019]. Disponible en: https://abdn.pure. elsevier.com/en/publications/eortc-qlq-c30-scoringmanual-3rd-edition

17. Yellen SB, Cella DF, Webster K, Blendowski C, Kaplan E. Measuring fatigue and other anemiarelated symptoms with the Functional Assessment of Cancer Therapy (FACT) measurement system. J Pain Symptom Manage. 1997;13(2):63-74. DOI: 10.1016/s0885-3924(96)00274-6

18. Latham NK, Bennett DA, Stretton CM, Anderson CS. Systematic review of progressive resistance 
strength training in older adults. J Gerontol A Biol Sci Med Sci. 2004;59(1):48-61. DOI: 10.1093/ gerona/59.1.m48

19. Serdà i Ferrer B-C. Calidad De Vida y Cáncer De Próstata: Efectos de un Programa de Ejercicio Físico. Revista Iberoamericana de Psicología del Ejercicio y el Deporte. 2011;6(1):13-22.

20. Segal RJ, Reid RD, Courneya KS, Malone SC, Parliament MB, Scott CG, et al. Resistance exercise in men receiving androgen deprivation therapy for prostate cancer. J Clin Oncol. 2003;21(9):1653-9. DOI: 10.1200/JCO.2003.09.534
21. Craike MJ, Livingston PM, Botti M. An exploratory study of the factors that influence physical activity for prostate cancer survivors. Support Care Cancer. 2011;19(7):1019-28. DOI: 10.1007/s00520-010-0929-3

22. Liou G-Y, Storz P. Reactive oxygen species in cancer. Free Radic Res. 2010;44(5):479-96. DOI: 10.3109/10715761003667554

23. Jemal Ahmedin, Siegel Rebecca, Ward Elizabeth, Hao Yongping, Xu Jiaquan, Murray Taylor, et al. Cancer Statistics, 2008. CA: A Cancer Journa for Clinicians. 2008;58(2):71-96. DOI: 10.3322/ CA.2007.0010
24. Bill-Axelson A, Holmberg L, Ruutu M, Garmo H, Stark JR, Busch C, et al. Radical prostatectomy versus watchful waiting in early prostate cancer. N Engl J Med. 2011;364(18):1708-17. DOI: 10.1056/ NEJMoa1011967

25. Veiga FG, Olmo JMC, Günthner S, Pallas MP, Moyano AS, Ramos EB, et al. [Development and validation of the first Spanish questionnaire of quality of life in patients with prostate cancer, based on the patient's perception. Actas Urol Esp. 2009;33(6):623-34. DOI: 10.1016/s0210-4806(09)74200-7 